\title{
Influencia del tratamiento térmico en las intercaras y propiedades mecánicas de un laminado multicapa de aluminio
}

\author{
C.M. Cepeda-Jiménez*, P. Hidalgo*, M. Pozuelo**, O.A. Ruano* y F. Carreño*
}

\begin{abstract}
Resumen
En este trabajo se han caracterizado la microestructura y las propiedades mecánicas alrededor de la intercara de un material multicapa de aluminio basado en las aleaciones Al 7075 y Al 2024. Las técnicas empleadas, principalmente, han sido EBSD y ensayos de cizalla para determinar propiedades mecánicas de las intercaras, en función del tiempo de solubilización durante el tratamiento térmico T6. Dicho tratamiento modifica la microestructura de las aleaciones constituyentes adyacentes a la intercara y, por tanto, las propiedades mecánicas de esta. La combinación de la ruta de procesado adecuada mediante laminación en caliente, junto con un tratamiento térmico optimizado conduce a intercaras resistentes, propensas a delaminar y, por ello, a materiales con propiedades mecánicas excelentes.
\end{abstract}

\section{Influence of the thermal treatment on the interfaces and mechanical properties of an aluminium multilayer laminate}

\begin{abstract}
The microstructure and mechanical properties in the interface region of a multilayer laminate based on high strength aluminium alloys, $\mathrm{Al} 7075$ and Al 2024, have been characterized mainly by EBSD and shear tests. It is shown that the time of solution treatment during T6 thermal treatment modifies the microstructure of the constituent alloys adjacent to the interface and, as a consequence, the interfacial mechanical properties. The combination of the correct rolling processing with optimized thermal treatment leads to strong interfaces prone to delaminate, and thus, materials with outstanding mechanical properties.
\end{abstract}

Keywords

Aluminium alloys; Thermomechanical processing; Mechanical properties; Interfaces; Microstructure.

\section{INTRODUCCIÓN}

La industria del aluminio ha realizado grandes esfuerzos para mejorar constantemente las propiedades mecánicas de las aleaciones de aluminio. Esto ha devenido en el desarrollo y posterior aplicación, en la industria aeroespacial, de aleaciones de muy alta resistencia como las de la serie $7 \mathrm{xxx}$ y alta tolerancia al daño como las de las series 2 xxx y $6 x x x$. Sin embargo, todavía, el gran reto es conseguir aleaciones con ambas propiedades mejoradas, es decir, alta resistencia y tenacidad.

Mediante el procesado de materiales laminados multicapa se pueden mejorar, simultáneamente, ambas propiedades mecánicas, que resultan muy superiores a las de sus materiales constituyentes de forma individual ${ }^{[1-4]}$.
El procesado de materiales multicapa mediante laminación en caliente es muy efectivo en la obtención de buenas uniones entre las distintas capas ${ }^{[5}$ y 6$]$. Además, simultáneamente, afina la microestructura. Los mecanismos de fractura que pueden tener lugar en las intercaras como, por ejemplo, la delaminación y posterior renucleación de nuevas grietas, son los responsables del aumento de tenacidad en materiales multicapa ${ }^{[7}$ y 8$]$. Por tanto, las propiedades mecánicas de las intercaras afectarán notablemente a las propiedades mecánicas globales del material multicapa ya que, estas, son numerosas y susceptibles de decohesión y deslizamiento ${ }^{[9]}$. La decohesión de la intercara será más probable cuando la resistencia a la fractura de la misma sea relativamente baja y los materiales adyacentes a ella tengan un elevado límite elástico. Esta condición es importante ya que

\footnotetext{
* Departamento de Metalurgia Física, CENIM, CSIC, Av. Gregorio del Amo 8, 28040 Madrid.

** Department of Materials Science and Engineering, University of California Los Angeles, E-V 2122D, 410 Westwood Plaza, Los Angeles, CA 90095, USA.
} 
materiales con facilidad para deformar plásticamente tenderán a ralentizar y dificultar la propagación de la grieta, evitando la delaminación en la intercara ${ }^{[10]}$.

Tras el procesado de laminación en caliente suele ser necesario realizar un tratamiento térmico para optimizar las propiedades mecánicas de las aleaciones constituyentes, las cuales deben su excelente resistencia mecánica al endurecimiento por precipitación. Estos tratamientos térmicos consisten en la solubilización de los elementos aleantes, seguido de temple en agua fría y, finalmente, un tratamiento de envejecimiento para conseguir una dispersión fina y homogénea de los precipitados endurecedores en el interior de los granos. En lo referente a los materiales multicapa, la región alrededor de las intercaras será de vital importancia, ya que variaciones en los tratamientos térmicos influirán en los procesos de difusión de elementos aleantes a través de las intercaras, en la microestructura de las aleaciones constituyentes y, por tanto, en sus propiedades mecánicas y, en último término, en las propiedades mecánicas de las intercaras y del material multicapa.

Por tanto, el objetivo de este trabajo es estudiar la influencia de diferentes tratamientos térmicos y, concretamente, de la etapa de solubilización debido a que, al realizarse a alta temperatura, $465^{\circ} \mathrm{C}$ para las aleaciones consideradas, es la etapa que más influirá en cambios microestructurales en las aleaciones constituyentes. Los resultados obtenidos mediante distintas técnicas de caracterización microstructural y mecánica de las aleaciones constituyentes sometidas a distintos tratamiento térmicos serán correlacionados con las propiedades mecánicas de las intercaras, caracterizadas mediante ensayos de cizalla.

\section{MÉTODO EXPERIMENTAL}

\subsection{Procesado}

Para el procesado del material compuesto multicapa se consideraron aleaciones de aluminio comerciales, como son $\mathrm{Al}$ 7075-T6 (Al-5.9wt\%Zn-2.5wt\%Mg, referenciada en este trabajo como "D") y Al 2024 (Al-4.4wt\%Cu-1.3wt\%Mg, referenciada como "L"). Las dimensiones de las chapas de partida fueron $150 \times 60 \times 2 \mathrm{~mm}^{3}$.

El material multicapa se realizó apilando 11 chapas alternadas de la aleación Al 7075 "D" (6 chapas), y de la aleación Al 2024 "L" (5 chapas). El apilamiento de chapas obtenido fue soldado mediante soldadura TIG, para evitar la entrada de oxígeno y el deslizamiento de las chapas durante la laminación. Posteriormente, dicho apilamiento fue laminado en caliente a $465^{\circ} \mathrm{C}$, temperatura a la cual se produce la solubilización de la aleación Al 7075. El procesado fue realizado en cinco ciclos de varias pasadas cada uno y una reducción del 4-8 \% por pasada, siendo recalentada la muestra a $465^{\circ} \mathrm{C}$, entre cada ciclo. La reducción en espesor total fue de 2,3:1, lo que corresponde a una deformación equivalente de $\varepsilon \sim 0,95$, según el criterio de von Mises. El material multicapa resultante se ha referenciado en este estudio como ADL11.

Debido a las altas temperaturas empleadas para el procesado y que, tras la laminación, el material multicapa enfría lentamente, fue necesario realizar un tratamiento térmico para recuperar la excelente resistencia mecánica de las aleaciones constituyentes. Además, previamente al tratamiento endurecedor T6, se realizó un recocido al laminado, de $6 \mathrm{~h}$ a $175^{\circ} \mathrm{C}$, para aliviar acritud y recuperar cierta ductilidad, y, así, evitar el fallo prematuro de las intercaras $^{[3 \text { y 11] }}$. Posteriormente, se realizó el tratamiento T6 y, en el presente estudio, se consideraron distintos tiempos de solubilización entre 2 y 30 min y se analizó su influencia sobre las propiedades del material multicapa.

\subsection{Caracterización microestructural}

La microestructura de las aleaciones de partida y contenidas en el material multicapa se analizaron en la sección LT (longitudinal-transversal), la cual contiene la dirección de laminación y la dirección normal al plano de laminación. La técnica empleada fue la difracción de electrones retrodispersados (EBSD) acoplada a un microscopio electrónico de barrido SEM-FEG JEOL JSM 6500F. Se prestó especial atención a la microestructura alrededor de las intercaras. Las fronteras de bajo ángulo definidas por una desorientación entre granos adyacentes de $2^{\circ}<\theta \leq 15^{\circ}$ se representaron mediante líneas blancas en los mapas de EBSD, mientras que las de alto ángulo $\left(\theta>15^{\circ}\right)$ se representaron mediante líneas negras. El espesor de los granos se determinó mediante el método de intercepción lineal en los mapas de EBSD, teniendo sólo en cuenta las fronteras de alto ángulo.

La preparación metalográfica para la caracterización mediante EBSD consistió en electropulido a $-15^{\circ} \mathrm{C}$ y $15 \mathrm{~V}$, utilizando como electrolito una disolución del 30 \% v. de $\mathrm{HNO}_{3}$ en $\mathrm{CH}_{3} \mathrm{OH}$.

La composición química alrededor de las intercaras se determinó mediante espectroscopía de energía dispersiva de rayos-X (EDX) y el programa Oxford Inca. 
INFLUENCIA DEL TRATAMIENTO TÉRMICO EN LAS INTERCARAS Y PROPIEDADES MECÁNICAS DE UN LAMINADO MULTICAPA DE ALUMINIO INFLUENCE OF THE THERMAL TREATMENT ON THE INTERFACES AND MECHANICAL PROPERTIES OF AN ALUMINIUM MULTILAYER LAMINATE

\subsection{Ensayo de microdureza Vickers}

Las medidas de microdureza se realizaron alrededor de las intercaras, en la sección LT, empleando un indentador Vickers con cargas de $100 \mathrm{~g}$ durante 15 s. Los valores de microdureza Vickers se representaron frente a la distancia a la intercara y se analizaron los gradientes de microdureza a través de la intercara, en función del tratamiento de solubilización considerado. La distancia desde el centro de cada huella de dureza a la intercara se midió mediante el programa de análisis de imagen SIGMA SCAN PRO.

\subsection{Ensayo de cizalla en las intercaras}

La resistencia de las intercaras, las cuales son las principales responsables de los mecanismos de fractura y de la mejora en tolerancia al daño del material multicapa, se midió mediante ensayos de cizalla. El ensayo se realizó en una máquina universal de ensayos Servosis, a una velocidad del travesaño de $0,005 \mathrm{~mm} / \mathrm{s}$, y se utilizaron probetas con unas dimensiones aproximadas de $10 \times 10 \times 3 \mathrm{~mm}^{3}$. El ensayo se realiza situando la probeta entre dos soportes metálicos (Fig. 1), de forma que la intercara que se va a ensayar se coloca sobresaliendo, justamente al borde del dispositivo de cizalla y paralela a la dirección de carga. Posteriormente, se utiliza un punzón de base cuadrada para la aplicación de la carga hasta que se produce la fractura de la intercara. La tensión de cizalla $(\tau)$ y la deformación de cizalla $(\gamma)$ se calcularon a partir de las siguientes expresiones ${ }^{[12]}$ :

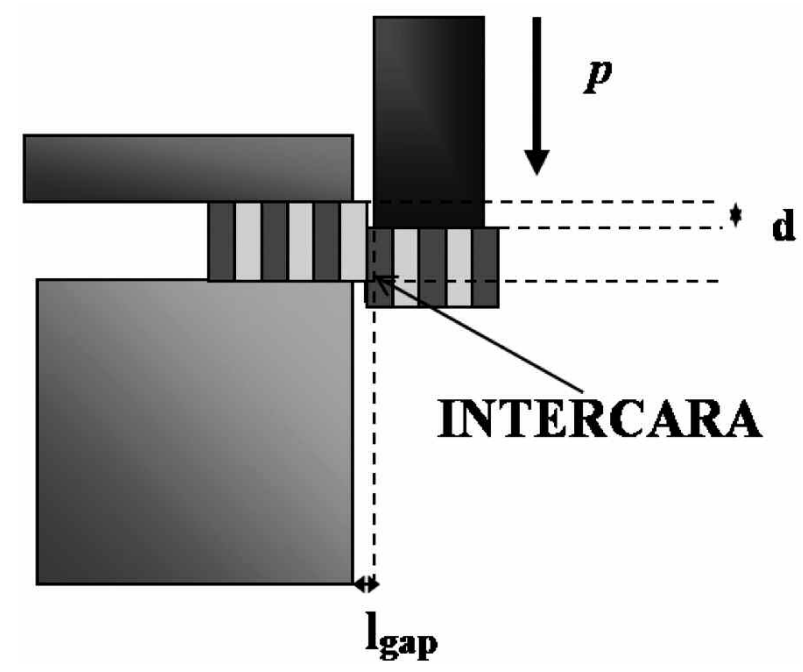

Figura 1. Esquema del dispositivo utilizado para la realización de los ensayos de cizalla.

Figure 1. Scheme of the shear test.

$$
\tau=\frac{p}{a e} \quad \gamma=\tan (\alpha)=\frac{d}{l_{\text {gap }}}
$$

donde, $a$ y $e$ son el ancho y espesor inicial de la muestra, respectivamente, $p$ es la carga aplicada sobre la muestra, $d$ es el desplazamiento del travesaño, $\alpha$ es el ángulo que forma la probeta respecto al soporte al cizallar y $l_{\text {gap }}$ es la distancia de separación entre la base fija y el punzón móvil que, en este caso, fue de 0,35 $\mathrm{mm}$.

\subsection{Ensayo de flexión en tres puntos}

Para caracterizar la respuesta mecánica de las intercaras y de las distintas capas, así como visualizar la propagación de la grieta frente a un impacto se realizaron ensayos de flexión en tres puntos, con probetas tipo Charpy $\left(10 \times 10 \times 55 \mathrm{~mm}^{3}\right)$ con entalla en $\mathrm{V}$ de $2 \mathrm{~mm}$ de profundidad. Los ensayos se realizaron sobre probetas en la orientación en serie, en la que la grieta propaga secuencialmente a través de las distintas capas y que es la disposición lógica del material multicapa en una posible aplicación en la industria aeroespacial. El ensayo se realizó en una máquina universal de ensayos Servosis, a una velocidad del travesaño de $0,04 \mathrm{~mm} / \mathrm{s}$. Los resultados obtenidos del ensayo se representan en curvas carga-desplazamiento.

\section{RESULTADOS Y DISCUSIÓN}

\subsection{Microestructura}

La microestructura de las aleaciones de partida en la orientación LT, representada mediante mapas de EBSD, se muestra en la figura 2. Los mapas se han coloreado de acuerdo a la leyenda incluida en la figura 2 a) y representan orientaciones cristalográficas paralelas a la dirección normal al plano de laminación (ND). El Al 7075 de partida (Fig. 2 a)) presenta una microestructura constituida de granos alargados en la dirección de laminación y un espaciado entre fronteras de alto ángulo en la dirección normal (ND), de 7,5 $\mu \mathrm{m}$. Por otra parte, el Al 2024 de partida (Fig. 2 b)) presenta una microestructura de granos recristalizados, menos elongados que en el Al 7075 y con un espaciado entre fronteras de alto ángulo en la dirección ND, de 7,4 $\mu \mathrm{m}$. En ambos materiales de partida se observan partículas grandes de compuestos intermetálicos ricos en hierro distribuidas al azar ${ }^{[13}$ y 14$]$.

La figura 3 muestra mapas de EBSD del material multicapa ADL11 alrededor de las intercaras, 

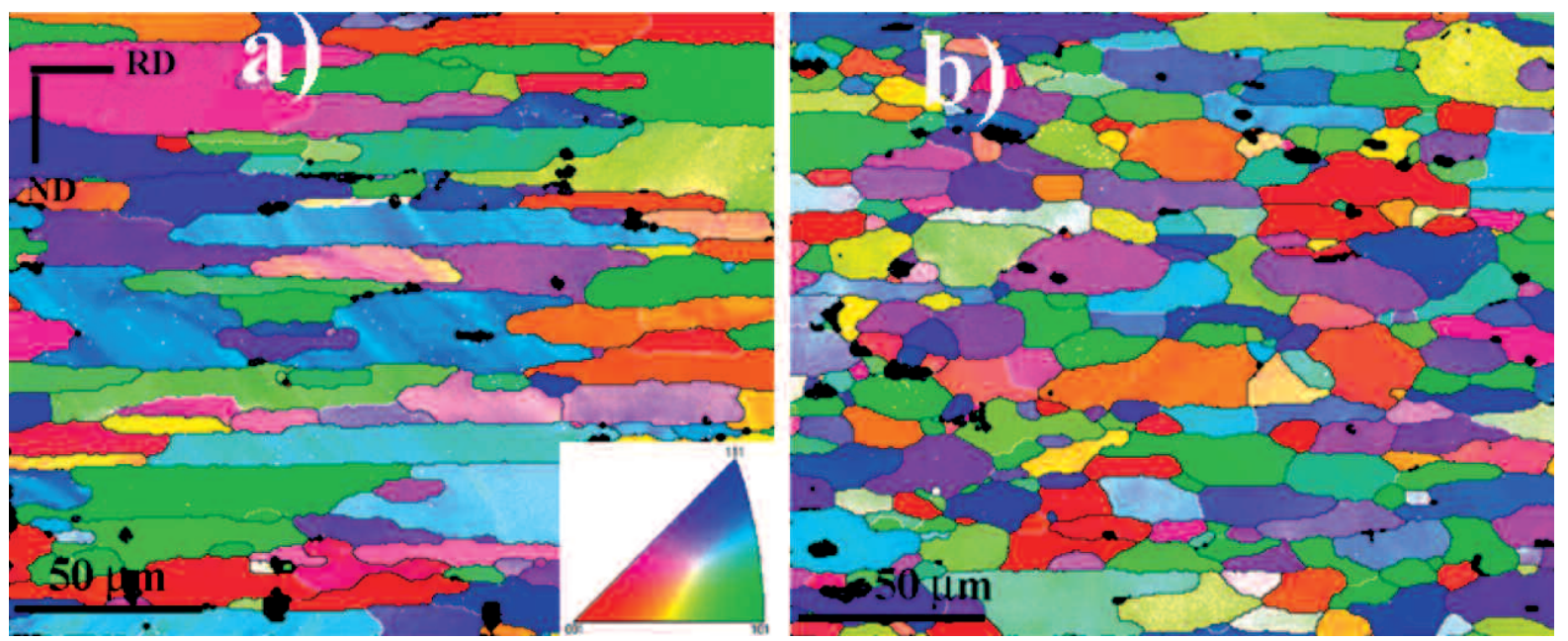

Figura 2. Mapas EBSD de la sección LT de las aleaciones de partida. a) Al 7075-T6; b) Al 2024-T3.

Figure 2. EBSD maps of LT sections of the as-received alloys: a) Al 7075-T6; b) Al 2024-T3.
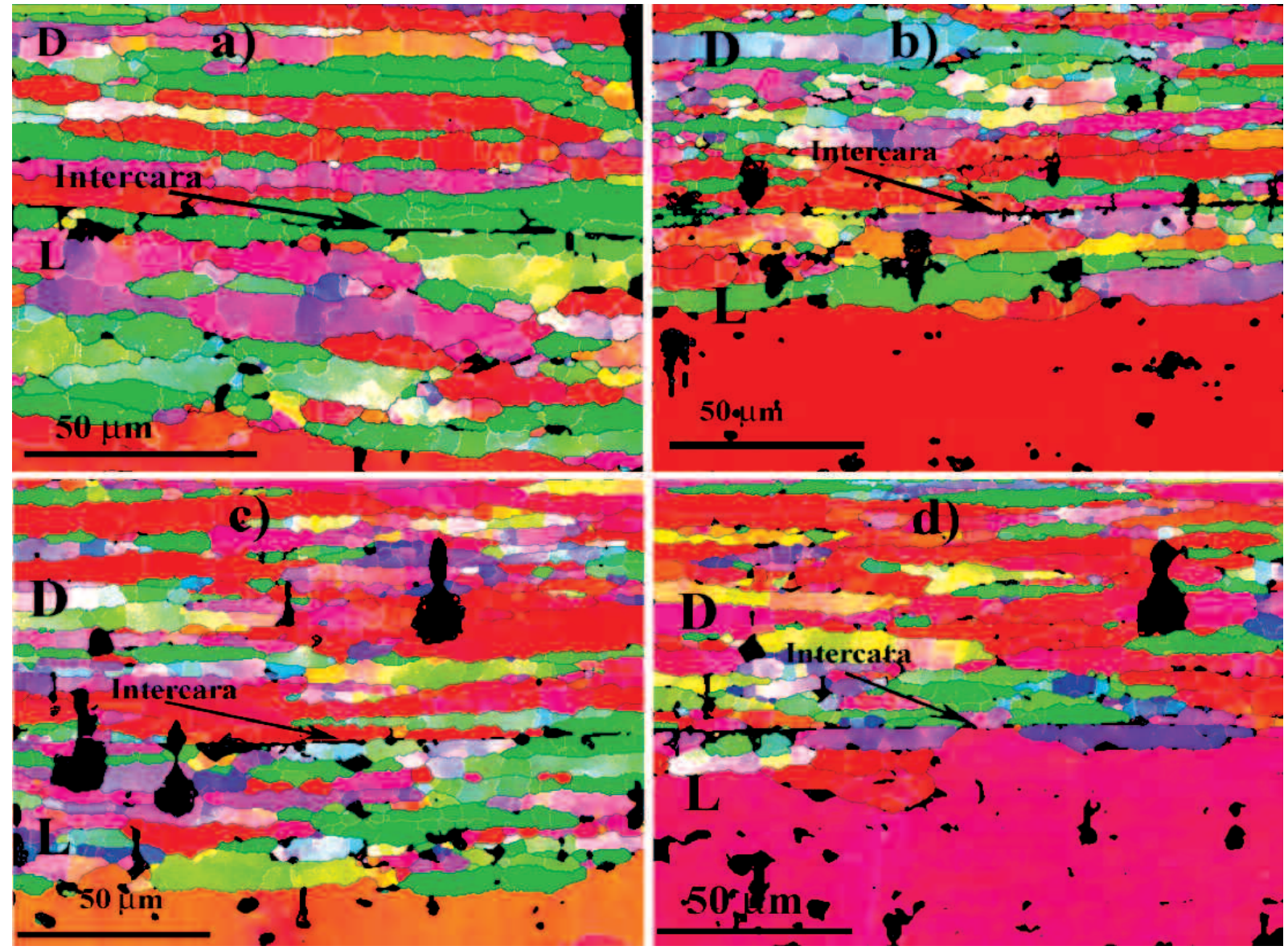

Figura 3. Mapas EBSD de secciones LT mostrando la microestructura de las aleaciones constituyentes alrededor de la intercara. a) Laminado ADL11 sin T6; b) ADL $11+465^{\circ} \mathrm{C} / 2 \mathrm{~min}$; c) ADL $11+465{ }^{\circ} \mathrm{C} / 5$ $\min$; d) ADL $11+465{ }^{\circ} \mathrm{C} / 30 \mathrm{~min}$.

Figure 3. EBSD maps of $L T$ sections of the aluminium alloys in the $A D L 11$ composite laminate close to the interface a) in the as-rolled state; and after different thermal treatments: b) $A D L 11+465{ }^{\circ} \mathrm{C} / 2$ min; c) $A D L 11+465^{\circ} \mathrm{C} / 5 \mathrm{~min}$; d) $A D L 11+465^{\circ} \mathrm{C} / 30 \mathrm{~min}$. 
INFLUENCIA DEL TRATAMIENTO TÉRMICO EN LAS INTERCARAS Y PROPIEDADES MECÁNICAS DE UN LAMINADO MULTICAPA DE ALUMINIO INFLUENCE OF THE THERMAL TREATMENT ON THE INTERFACES AND MECHANICAL PROPERTIES OF AN ALUMINIUM MULTILAYER LAMINATE

después del tratamiento de recocido $\left(175^{\circ} \mathrm{C} / 6 \mathrm{~h}\right)$ y sin tratamiento T6 (Fig. 3 a)) o sometido a distintos tiempos de solubilización a $465^{\circ} \mathrm{C}$ durante el tratamiento T6: 2 min (Fig. 3 b)), 5 min (Fig. 3 c)) y 30 min (Fig. 3 d)). Estos mapas EBSD ponen de manifiesto la importancia del tiempo a temperatura de solubilización, sobre la evolución de la microestructura. En todos los mapas se puede ver la intercara en el centro de la imagen, siendo la aleación de la parte superior del mapa, Al 7075 y, la de la parte inferior, Al 2024. Las partículas de alúmina presentes en la intercara se encuentran homogénea y continuamente distribuidas a lo largo de la misma.

Durante el procesado de laminación en caliente, la matriz de aluminio es capaz de deformar plásticamente; sin embargo, la alúmina, que es muy frágil, responde a la tensión generada en la intercara fracturándose. Por tanto, el aluminio con mayor plasticidad ocupa los huecos creados entre las partículas fracturadas de alúmina dando lugar a la unión de las dos capas metálicas ${ }^{[15]}$. Además, ocurrirá difusión de elementos aleantes entre las dos capas, fruto de la deformación y la temperatura del procesado.

El Al 7075-D, tras el procesado (Fig. 3 a)), muestra una microestructura de granos muy alargados en la dirección de laminación y separados por fronteras de alto ángulo. Esta microestructura se conserva después de los distintos tratamientos térmicos considerados (Fig. 3 b)-d)). En general, en la dirección normal (ND), las fronteras de grano son de bajo ángulo, observando una subestructura en el interior de los granos alargados. Tras el procesado, el espaciado entre fronteras de alto ángulo en la dirección ND se reduce a 4,5 $\mu \mathrm{m}$ y se observa un ligero incremento con el tiempo de solubilización, desde 4,1 $\mu \mathrm{m}$ para $2 \mathrm{~min}$ hasta 4,6 $\mu \mathrm{m}$ para $30 \mathrm{~min}$. Este crecimiento normal de grano se asocia con la restauración de las estructuras fuera de equilibrio, la aniquilación de defectos y la relajación parcial de tensiones residuales ${ }^{[16]}$.

Por otro lado, el Al 2024 muestra una microestructura de granos más finos que la de partida y alargados en la dirección de laminación, sólo cerca de la intercara ya que lejos de ésta se observa un crecimiento anormal de grano, el cual progresa hacia la intercara conforme aumenta el tiempo de solubilización a $465^{\circ} \mathrm{C}$ durante el tratamiento T6. De esta observación se puede deducir que las partículas constituyentes y los precipitados presentes en $\mathrm{Al} 2024$ no fijan la microestructura a alta temperatura. También, se puede observar en los mapas EBSD, que la actividad de migración de las fronteras de grano se frena en la intercara (Fig. 3 d)), lo cual se podría atribuir a las partículas de alúmina, cuyo tamaño y distribución a través de la intercara consigue impedir esta migración de fronteras de grano.

Por otro lado, fruto de la tensión, deformación plástica, alta temperatura y gradientes de concentración se produce una amplia zona de difusión de elementos aleantes a través de la intercara (Fig. 4). Esta difusión de elementos aleantes, los cuales dan lugar a los precipitados endurecedores en este tipo de aleaciones, determinará las propiedades mecánicas de las aleaciones constituyentes en las cercanías de la intercara. La figura 4 muestra los gradientes de concentración de zinc (Fig. 4 a)) y cobre (Fig. 4 b)), que son
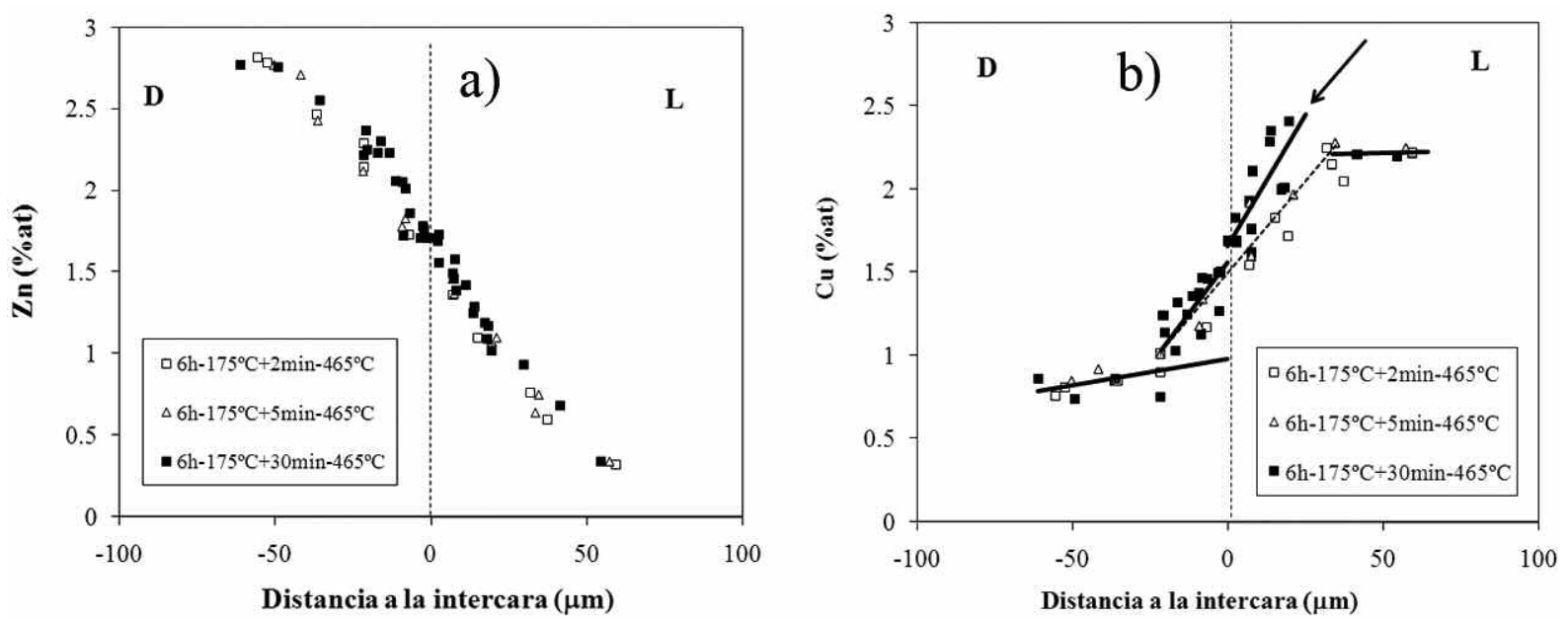

Figura 4. Porcentaje atómico de a) Zn y b) Cu alrededor de distintas intercaras del laminado ADL11, en función de distintos tiempos de solubilización durante el tratamiento T6.

Figure 4. Atomic percent of a) $\mathrm{Zn}$ and b) Cu composition across different interfaces (plotted in a single curve) in the ADL11 composite laminate after different thermal treatments. 
los principales elementos aleantes en las aleaciones consideradas.

Para ambos elementos, de forma general, el ancho de la región de difusión es 30-50 $\mu \mathrm{m}$ a ambos lados de la intercara. La extensión de la difusión del zinc (Fig. 4 a)) no parece estar influenciada por el tiempo de solubilización. En cambio, la región con gradiente de concentración del cobre (Fig. 4 b)) es más amplia para tiempos de solubilización más cortos. Este comportamiento puede resultar inesperado ya que tiempos prolongados (30 $\mathrm{min}$ ) a alta temperatura serían favorables para aumentar la distancia de difusión. Además, para el tiempo más largo, de 30 min, se observan distintas pendientes en el gradiente de concentración de cobre, a ambos lados de la intercara, siendo más pronunciada en el lado del Al 2024. Por tanto, los resultados muestran que el gradiente de concentración de cobre en el Al 2024 tiende a ser igual a la distancia donde todavía se observan granos finos, antes de migrar las fronteras de grano hacia la intercara con el aumento del tiempo de solubilización. Este estrecho gradiente de concentración de cobre en el Al 2024, para un tiempo de solubilización de $30 \mathrm{~min}$, se debe al arrastre de átomos de cobre hacia la intercara, junto con el anormal crecimiento de grano observado

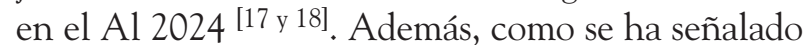
mediante una flecha en la figura 4 b), para esta condición de tratamiento (30 min), cerca de la intercara se observa una concentración de cobre ligeramente superior a la del material masivo lejos de la intercara, lo que indicaría que se ha producido una ligera acumulación de átomos de cobre en esta zona, fruto de ese arrastre de soluto ya comentado.

\subsection{Ensayo de microdureza Vickers}

La difusión gradual de elementos aleantes a través de la intercara crea, por tanto, una región con gradiente en propiedades mecánicas, fruto de la distinta concentración de precipitados endurecedores (Fig. 5).

Las líneas punteadas horizontales, en la figura 5, corresponden a los valores de dureza de las aleaciones de partida Al 7075 (188 HV) y Al 2024 (138 HV). Tras el procesado de laminación en caliente, a $465^{\circ} \mathrm{C}$, la dureza de las aleaciones constituyentes disminuye de forma acusada, siendo $92 \mathrm{HV}$ para Al 7075 y 120 HV para Al 2024, debido al lento enfriamiento del material multicapa después del procesado.

Todos los tratamientos T6 considerados aumentan notablemente la dureza del Al 7075 presente en el compuesto multicapa, siendo superior al valor de partida. Cabe destacar que las temperaturas de T6 seleccionadas son óptimas para el Al 7075, que es la aleación de mayor resistencia presente en el laminado.

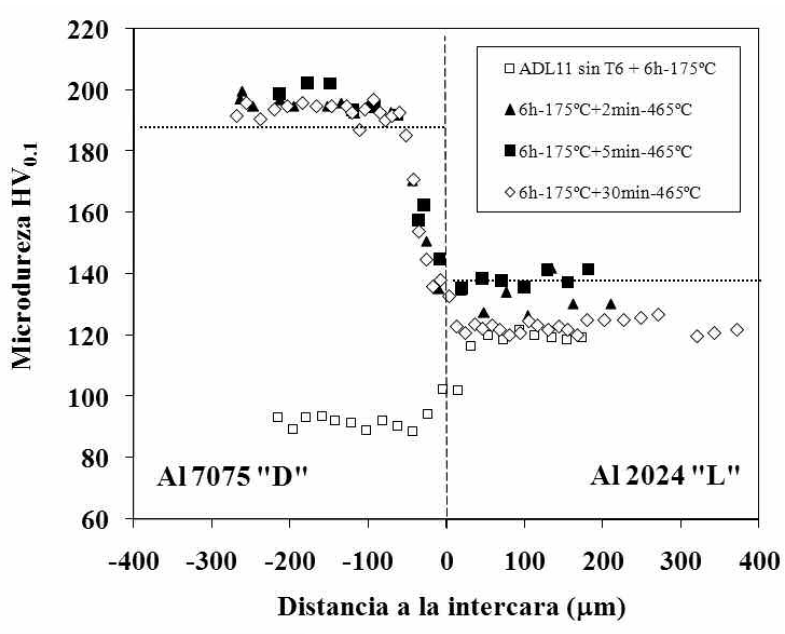

Figura 5. Microdureza Vickers (100 g/15 s) alrededor de diferentes intercaras del laminado ADL11, en función de distintos tiempos de solubilización durante el tratamiento T6.

Figure 5. Microhardness Vickers (100g/15s) across different interfaces (plotted in a single curve) in the ADL11 composite laminate after different thermal treatments.

Aún así, un tiempo de 5 min de solubilización produjo en el Al 2024 un valor de dureza similar al de partida. En ambas aleaciones, el máximo de dureza se produjo para un tiempo de solubilización de $5 \mathrm{~min}$ y, un incremento de tiempo hasta $30 \mathrm{~min}$, produce una pequeña disminución en la dureza debido al ligero aumento de tamaño de grano.

\subsection{Ensayo de cizalla en las intercaras}

Para cuantificar las propiedades mecánicas de las intercaras (resistencia y ductilidad), éstas se sometieron a ensayos de cizalla en función de distintos tratamientos térmicos. Los resultados obtenidos, promedio de al menos 6 intercaras de diferentes muestras con el mismo tratamiento térmico, se incluyen en la tabla I. El objetivo es correlacionar la resistencia y ductilidad, por tanto la tenacidad de las intercaras, con la microestructura y propiedades mecánicas de las aleaciones adyacentes a dichas intercaras. Por comparación, también se han incluido en la tabla I ciertas propiedades mecánicas bajo tensión de cizalla de las aleaciones constituyentes de partida.

La tensión máxima de cizalla del Al 7075 y Al 2024 es 279 y $236 \mathrm{MPa}$, respectivamente, y la deformación plástica de cizalla es 0,8 y 1,2. En cuanto al laminado ADL11, en todos los ensayos de cizalla realizados, la 
INFLUENCIA DEL TRATAMIENTO TÉRMICO EN LAS INTERCARAS Y PROPIEDADES MECÁNICAS DE UN LAMINADO MULTICAPA DE ALUMINIO INFLUENCE OF THE THERMAL TREATMENT ON THE INTERFACES AND MECHANICAL PROPERTIES OF AN ALUMINIUM MULTILAYER LAMINATE

Tabla I. Propiedades mecánicas de las intercaras en el material multicapa laminado ADL11 obtenidas mediante ensayos de cizalla, en función de distintos tratamientos térmicos. $\left(\tau_{\max }=\right.$ tensión máxima de cizalla; $\tau_{Y S}=$ límite elástico; $\gamma_{\text {plastic max }}=$ deformación plástica de cizalla máxima; $A=a$ rea bajo la curva de cizalla)

Table I. Mechanical properties of interfaces in the ADL11 composite laminate from shear tests as a function of different thermal treatments $\left(\tau_{\max }=\right.$ maximum shear strength; $\tau_{Y S}=$ yield point; $\gamma_{\text {plastic max }}=$ maximum plastic shear strain; $A=$ area under shear load-displacement curve)

\begin{tabular}{|c|c|c|c|c|}
\hline Material y tratamiento & $\tau_{\max }(\mathrm{MPa})$ & $\tau_{Y S}(\mathrm{MPa})$ & $\gamma_{\text {plastic max. }}$ & $\mathrm{A}\left(\mathrm{kJ} / \mathrm{m}^{2}\right)$ \\
\hline Al 7075-T6 “D” & 279 & 212 & 0.82 & 96 \\
\hline Al 2024-T3 “L” & 236 & 154 & 1.2 & 101 \\
\hline ADL $11+6 \mathrm{~h}-175{ }^{\circ} \mathrm{C}$ & 107 & 37 & 0.34 & 10.1 \\
\hline $\mathrm{ADL} 11+6 \mathrm{~h}-175^{\circ} \mathrm{C}+2 \min -465^{\circ} \mathrm{C}$ & 111 & 56 & 0.19 & 7.4 \\
\hline ADL11 + $6 \mathrm{~h}-175^{\circ} \mathrm{C}+5 \min -465^{\circ} \mathrm{C}$ & 115 & 60 & 0.23 & 9.1 \\
\hline $\mathrm{ADL} 11+6 \mathrm{~h}-175^{\circ} \mathrm{C}+30 \min -465^{\circ} \mathrm{C}$ & 129 & 51 & 0.33 & 12.8 \\
\hline
\end{tabular}

fractura se produjo en el punto de máxima resistencia, con instantánea decohesión de la intercara. En general, las curvas de cizalla (no incluidas) mostraron cierta deformación plástica previamente a la fractura de la intercara. Los resultados de los ensayos de cizalla incluidos en la tabla I muestran cierta influencia del tratamiento térmico sobre la resistencia y ductilidad interfacial. En general, las intercaras del laminado son bastante frágiles, con valores de resistencia máxima comprendidos entre $107 \mathrm{MPa}$ para el laminado sin T6 y $129 \mathrm{MPa}$ para el tratado, con 30 min de tiempo de solubilización. Por otro lado, la deformación plástica máxima de cizalla oscila entre 0,19 para el laminado ADL11 solubilizado 2 min y 0,34 para el material sin tratar. Hay que destacar que las resistencias a la cizalla requeridas para uniones en estructuras aeroespaciales son generalmente más bajas (10-20 MPa) que las obtenidas en el presente estudio ${ }^{[19]}$. La tenacidad a cizalla de las intercaras se calculó como el área bajo la curva carga-desplazamiento en un ensayo de cizalla. La tenacidad interfacial promedio oscila entre $7,4 \mathrm{~kJ} / \mathrm{m}^{2}$ para el laminado ADL11 tratado con un tiempo de solubilización de $2 \min$ y $12,8 \mathrm{~kJ} / \mathrm{m}^{2}$ para el material solubilizado durante $30 \mathrm{~min}$. Las intercaras de las muestras tratadas térmicamente (T6) mostraron mayor límite elástico y resistencia máxima que en el caso del material sin tratar, como consecuencia del endurecimiento por precipitación. Además, el mayor valor de límite elástico corresponde al laminado solubilizado durante $5 \mathrm{~min}$, estando de acuerdo con los valores de microdureza más altos observados también para esta condición de tratamiento térmico.

Las intercaras en el laminado ADL11 sin tratamiento térmico mostraron valores más bajos de límite elástico que las de los laminados tratados. Esto se debe a la disminución notable de dureza que experimentan las aleaciones constituyentes, como fruto del lento enfriamiento tras el procesado y la formación de gruesos precipitados en fronteras de grano. Esta más baja resistencia, sin embargo, irá asociada con mayor ductilidad y capacidad para acomodar la deformación, lo que se refleja en un valor de tenacidad interfacial comparable a los obtenidos para el laminado solubilizado 5 min y que ha alcanzado el pico de dureza.

Por otro lado, el material tratado con un tiempo de solubilización de 2 min presenta el valor promedio de tenacidad interfacial más bajo. Esto se debe a una más alta densidad de defectos, como fronteras de grano asociadas al tamaño de grano más fino, o número de dislocaciones debido a un tiempo más corto de solubilización a alta temperatura, que disminuye la capacidad para acomodar la deformación y, en consecuencia, la ductilidad y tenacidad de la intercara ${ }^{[20]}$. De hecho, los tiempos de tratamiento más cortos, 2 y 5 min, dieron lugar a tamaños de grano más finos, dureza Vickers más alta y mayor gradiente de concentración, esto es., mayor área endurecida, que el laminado sometido a un tratamiento de solubilización más prolongado, de $30 \mathrm{~min}$. Estos resultados indican la correlación entre las propiedades mecánicas de los materiales adyacentes a la intercara y su capacidad para acomodar la deformación, con la respuesta mecánica de la intercara. Por tanto, para una intercara dada, la evolución de la resistencia y ductilidad en función del tratamiento térmico está controlada por el límite elástico de las aleaciones adyacentes a la intercara y éste, a su vez, por el endurecimiento por precipitación y el tamaño de grano, resultado del tratamiento térmico considerado. Materiales adyacentes a la intercara más resistentes y frágiles, los cuales acomoden peor la deformación, darán lugar a intercaras menos 
tenaces. Finalmente, el comportamiento mecánico relativo intercara/aleaciones adyacentes determinará la capacidad de la intercara para delaminar y, por tanto, las propiedades mecánicas globales del material multicapa. Esto se verá claramente, a continuación, en los ensayos de flexión en tres puntos.

\subsection{Ensayo de flexión en tres puntos}

La figura 6 muestra curvas carga-desplazamiento obtenidas a partir de ensayos de flexión en tres puntos. La comparación del área bajo las curvas cargadesplazamiento permite comparar la tenacidad de los distintos materiales. Se realizaron ensayos de flexión para las aleaciones de partida, para el laminado multicapa sin tratamiento térmico y para el sometido a tratamiento T6 con un tiempo de solubilización de $30 \mathrm{~min}$. En ambos casos, la tenacidad interfacial promedio fue similar (Tabla I); sin embargo, la resistencia mecánica de las aleaciones adyacentes es notablemente diferente, lo que se verá claramente reflejado en el comportamiento de las intercaras durante el ensayo de flexión en tres puntos. Los materiales de partida muestran alta resistencia a la flexión: $960 \mathrm{MPa}$ el Al 7075 y $750 \mathrm{MPa}$ el Al 2024. Sin embargo, presentan baja ductilidad. Por otro lado, el laminado ADL11 sin tratamiento térmico muestra baja resistencia $(520 \mathrm{MPa})$ debido

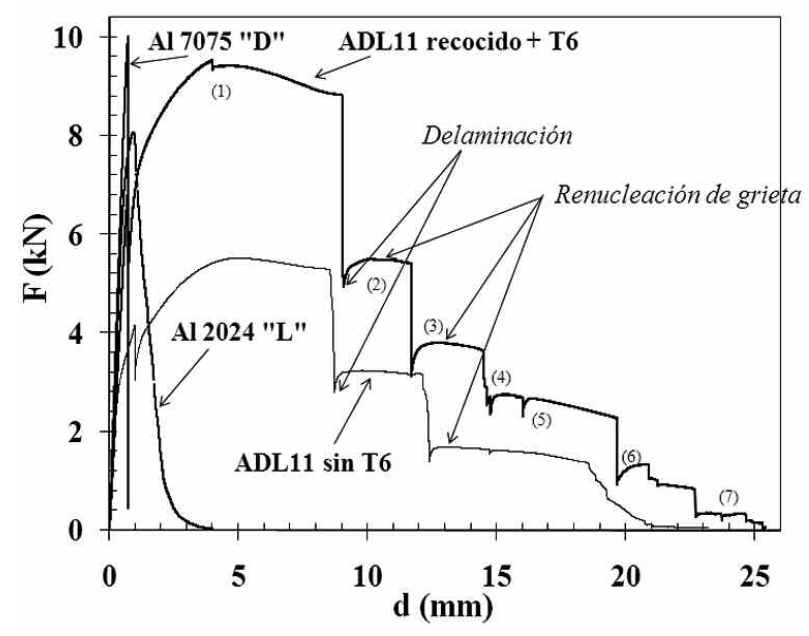

Figura 6. Curvas carga-desplazamiento obtenidas mediante ensayos de flexión en tres puntos de las aleaciones constituyentes de partida, y del laminado ADL11 con y sin tratamiento térmico T6.

Figure 6. Three-point bend test of as-received aluminum alloys and of as-rolled ADL11 and T6treated ADL11 composite laminates. a las altas temperaturas alcanzadas durante el procesado y el lento enfriamiento a temperatura ambiente. Por tanto, de nuevo, se pone de manifiesto la necesidad de realizar un tratamiento térmico tras el procesado. En contraste, el laminado ADL11 sometido a tratamiento T6 muestra un máximo de resistencia a la flexión de $890 \mathrm{MPa}$, más alto que el valor esperado según la regla de las mezclas, y una ductilidad excelente.

Por otro lado, las curvas de flexión incluidas en la figura 6 muestran un patrón de fractura similar aunque se observan claras diferencias entre ellas. La forma escalonada de las curvas indica que están operando mecanismos extrínsecos de tenacidad, en este caso, delaminaciones en las intercaras. Ambas curvas muestran caídas de carga debido a la fractura de diferentes capas, hasta que la grieta es frenada en la correspondiente intercara. También, se pueden observar regiones planas después del frenado de la grieta, correspondientes a la deformación plástica del material restante, hasta que se produce una nueva caída de carga, lo que indica que se ha alcanzado la deformación crítica necesaria para la renucleación de una nueva grieta. Además, se puede observar que la curva de flexión correspondiente al laminado tratado térmicamente muestra un mayor número de caídas de carga (aprox. 8), que el laminado sin tratamiento térmico. Esto indica que en el material tratado se han producido, prácticamente, tantas delaminaciones como intercaras contiene el laminado, cosa que no ocurre en el material sin tratar, con reducido número de delaminaciones. Este diferente número de delaminaciones se observa claramente en la figura 7 , la cual muestra dos macrografías del laminado ADL11 sin tratamiento térmico (Fig. 7 a)) y sometido a tratamiento T6-30min (Fig. 7 b)) después del ensayo de flexión.

Este diferente comportamiento mecánico se debe, como ya se ha comentado anteriormente, al comportamiento relativo intercara/aleaciones adyacentes, que en el caso del material sin tratar, debido a la baja resistencia mecánica de las aleaciones constituyentes, permite acomodar mejor la deformación, reduciendo la fuerza impulsora de la grieta y, en último término, evitando la delaminación de la intercara. Por el contrario, tras el tratamiento T6, la alta resistencia y menor ductilidad de las aleaciones adyacentes a la intercara acelera la propagación de la grieta, favoreciendo la delaminación en cada una de las intercaras.

Finalmente, hay que destacar el extraordinario aumento del área bajo la curva carga-desplazamiento (tenacidad) que produce el gran número de delaminaciones en el laminado ADL11-T6 respecto a las aleaciones de partida. Se ha mostrado, mediante ensayos 

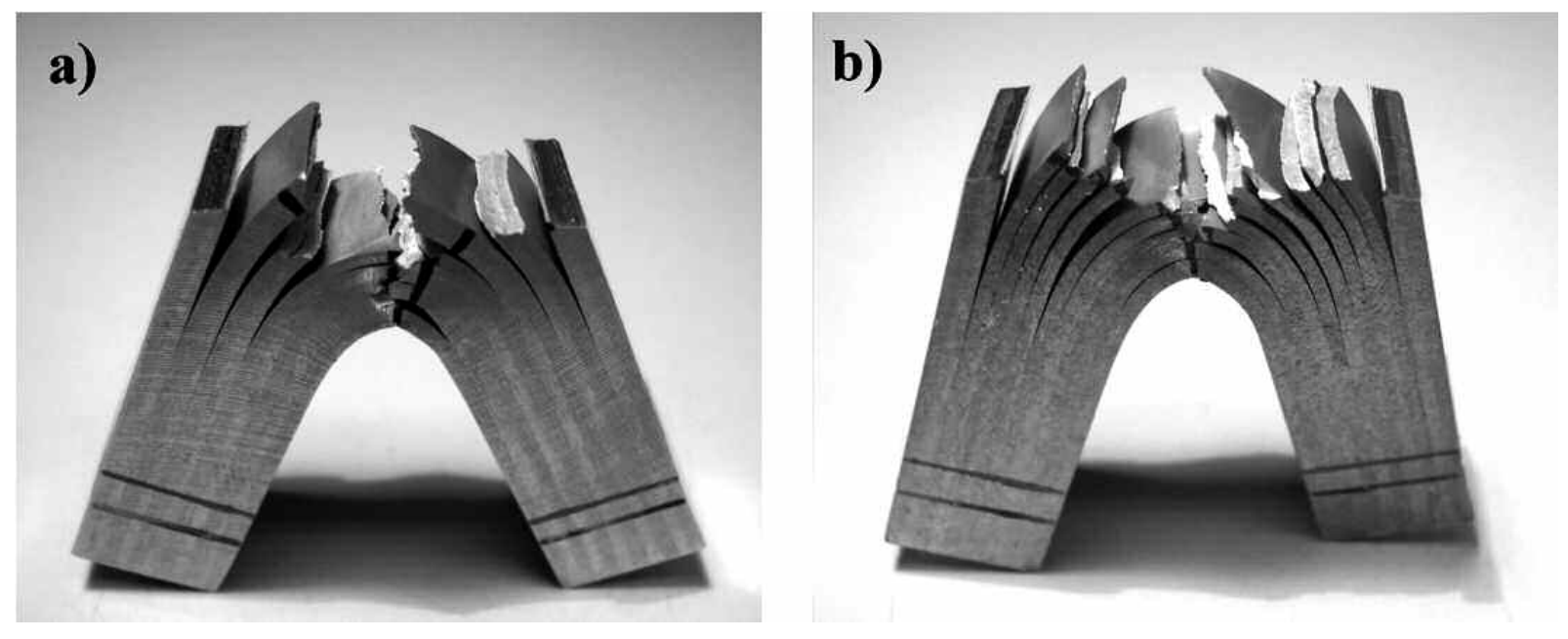

Figura 7. Macrografías de probetas ensayadas mediante ensayos de flexión en tres puntos del laminado ADL11: a) sin tratamiento T6; b) con tratamiento T6.

Figure 7. Macrographs of bend-test fractured composite laminate samples: (a) as-rolled ADL11 composite laminate, (b) T6-treated.

de impacto Charpy, que la tenacidad del material multicapa ADL11+T6 fue 21 y 7 veces superior a la del Al7075 y Al 2024 de partida, respectivamente ${ }^{[3]}$, demostrando tanto la efectividad de la configuración de aleaciones elegida como del procesado y tratamiento T6 realizados.

\section{CONCLUSIONES}

En el presente trabajo se han caracterizado la microestructura y las propiedades mecánicas interfaciales de un material multicapa laminado, compuesto por las aleaciones de alta resistencia $\mathrm{Al}$ 7075 y Al 2024.

La variación de la tenacidad interfacial en función del tiempo de solubilización durante el tratamiento T6 se debe a la combinación de resistencia y ductilidad de las aleaciones constituyentes del laminado adyacentes a la intercara, las cuales deben acomodar la deformación durante el ensayo de cizalla.

El comportamiento mecánico relativo intercara/aleaciones adyacentes determinará la respuesta mecánica de la intercara y su propensión a delaminar y, por tanto, las propiedades mecánicas globales del material multicapa procesado.

El procesado adecuado junto con el tratamiento térmico correcto permite obtener materiales multicapa de aluminio con extraordinarias propiedades mecánicas.

\section{Agradecimientos}

Los autores agradecen al MICINN la financiación recibida a través de los proyectos MAT2006-11202 y MAT2009-14452.

\section{REFERENCIAS}

[1] M.E. Launey y R.O. Ritchie, Adv. Mater. 21 (2009) 1-8.

[2] C.M. Cepeda-Jiménez, M. Pozuelo, O.A. Ruano y F. Carreño, Mater. Sci. Eng. A 490 (2008) 319-327.

[3] C.M. Cepeda-Jiménez, M. Pozuelo, J.M. GarcíaInfanta, O.A. Ruano y F. Carreño, Metall. Mater. Trans. A 40 (2009) 69-79.

[4] C.M. Cepeda-Jiménez, P. Hidalgo, M. Pozuelo, O.A. Ruano y F. Carreño, Metall. Mater. Trans. A 41 (2010) 61-72.

[5] M.Z. Quadir, A. Wolz, M. Hoffman y M. Ferry, Scripta Mater. 58 (2008) 959-962.

[6] G.P. Chaudhari y V. Acoff, Compos. Sci. Technol. 69 (2009) 1.667-1.675.

[7] J. Zhang y J.J. Lewandowski, J. Mater. Sci. 29 (1994) 4.022-4.026.

[8] S. Nambu, M. Michiuchi, J. Inoue y T. Koseki, Compos. Sci. Technol. 69 (2009) 1.936-1.941.

[9] A.G. Evans y J.W. Hutchinson, Acta Metall. Mater. 43 (1995) 2.507-2.530.

[10] M.S. Hu y A.G. Evans, Acta Metall. 37 (1989) 917-925. 
[11] N. Kamikawa, N. Tsuji, X. Huang y N. Hansen, Acta Mater. 54 (2006) 3.055-3.066.

[12] G.E. Dieter, Mechanical Metallurgy, SI Metric Edition, $3^{\text {rd }}$ edition, McGraw-Hill Book Co., Londres, Inglaterra, 1988, pp. 12-15.

[13] C.E. Campbell, L.A. Bendersky, W.J. Boettinger y R. Ivester, Mater. Sci. Eng. A 430 (2006) 15-26.

[14] Y. Xue, H. El Kadiri, M.F. Horstemeyer, J.B. Jordon y H. Weiland, Acta Mater. 55 (2007) 1.975-1.984.

[15] C.Y. Barlow, P. Nielsen y N. Hansen, Acta Mater. 52 (2004) 3.967-3.972.
[16] R.K. Islamgaliev, N.F. Yunusova, I.N. Sabirov, A.V. Sergueeva y R.Z. Valiev, Mater. Sci. Eng. A 319-321 (2001) 877-881.

[17] J.P. Lokker, A.J. Böttger, W.G. Sloof, F.D. Tichelaar, G.C.A.M. Janssen y S. Radelaar, Acta Mater. 49 (2001) 1.339-1.349.

[18] J. Dennis, P.S. Bate y F.J. Humphreys, Acta Mater. 57 (2009) 4.539-4.547.

[19] Y. Huang, N. Ridley, F.J. Humphreys y J.-Z. Cui, Mater. Sci. Eng. A 266 (1999) 295-302.

[20] J.W. Hutchinson y A.G. Evans, Acta Mater. 48 (2000) 125-135. 